\title{
SCIROCCO+: SIMULATION CODE OF INTERFEROMETRIC-OBSERVATIONS FOR ROTATORS AND CIRCUMSTELLAR OBJECTS INCLUDING NON-RADIAL PULSATIONS
}

\author{
M. Hadjara ${ }^{1,2}$, F. Vakili², A. Domiciano de Souza ${ }^{2}$, F. Millour ${ }^{2}$, \\ R. Petrov ${ }^{2}$, S. Jankov ${ }^{3}$ and P. Bendjoya ${ }^{2}$
}

\begin{abstract}
The VLTI (Very Large Telescope Interferometer) makes available milli-arcsecond-scale observations in the infrared. It offers new possibilities for constraining stellar structures such as polar jets, equatorial disks and rotationally-flattened photospheres of Be stars. Such constraints allows us to better estimate the stellar fundamental parameters and refine the mechanisms such as mass loss, pulsation and magnetism that govern the variability and evolution of these stars.

In this paper we present a chromatic semi-analytical model of fast rotators, which allows us to study the dynamics and the interaction between the photosphere and the wind of fast rotating stars of $\mathrm{O}, \mathrm{B}$, $\mathrm{A}$ and $\mathrm{F}$ spectral types. Our simple analytical model addresses the oblateness, inclination and position angle of the rotation axis of the star. It produces iso-velocity maps and intensity maps. It includes line profiles, limb-darkening and the von Zeipel effect and the non-radial pulsations.

SCIROCCO+: Simulation Code of Interferometric-observations for ROtators and CirCumstellar Objects including Non-Radial Pulsations, includes all the parameters cited above in order to be fast, powerful and light simulation tool in high angular resolution of rotating objects.
\end{abstract}

\footnotetext{
${ }^{1}$ Centre de Recherche en Astronomie, Astrophysique et Géophysique (CRAAG), Route de l'Observatoire, BP. 63, Bouzareah, 16340 Alger, Algérie; e-mail: m.hadjara@craag.dz

${ }^{2}$ Laboratoire J.-L. Lagrange, UMR 7293, Observatoire de la Côte d'Azur (OCA), Université de Nice-Sophia Antipolis (UNS), Centre National de la Recherche Scientifique (CNRS), Campus Valrose, 06108 Nice Cedex 2, France; e-mail: Massinissa.Hadjara@oca.eu

${ }^{3}$ Astronomical Observatory of Belgrade, Volgina 7, PO Box 74, 11060 Belgrade, Serbia
} 


\section{Introduction}

The Be stars with low metallicity are supposed to produce little or no magnetic field. This absence of magnetic field leads to a high spin-up during the contraction then formation of these stars (Martayan et al. 2006). This rotation rate can attain more than $80 \%$ of the critical, or breakup, velocity $v_{c}=\sqrt{\left(G M / R_{c}\right)}$ (with $R_{c}$ the equatorial radius at this velocity) in some cases. These fast-rotating stars are called "fast rotators" and exhibit a number of peculiar characteristics (Domiciano de Souza et al. 2003), among which geometrical flattening, coupled with gravitational darkening von Zeipel (1924), making the poles hotter than the equator.

The models from Collins \& Sonneborn (1977) indicate a two-components spectral energy distribution (SED) for these stars, with an infrared excess due to gravity darkening. Hence, it is not easy to place these stars in one single spectral classification, as the observed SED depends on its rotational velocity and inclination angle (Maeder \& Peytremann 1972).

Furthermore, rapid rotation induce an additional change in the apparent spectral type and class of the star (Collins \& Harrington 1966). Indeed, the full widths at half-maximum (FWHM) of UV lines are generally narrower (up $0.2 \mathrm{~km} / \mathrm{s}$ ) than those of the visible lines due again to gravitational darkening (Hutchings et al. 1979), since the spectral lines, depending on the temperature and gravity, are not formed uniformly on the star. This has an impact on the estimate of the inclination angle (Hutchings \& Stoeckley 1977) and, hence, the estimation of the spectral type of the star. A classification based on the spectral ratio between the widths of these lines would be distorted by this effect (Collins 1974).

In addition, mechanisms such as meridional circulation and/or turbulence may affect the internal structure of the star and its evolution (Meynet 2009). Thus, fast rotators have always been considered as a physics laboratory to study stellar interiors, stellar evolution and primordial stars.

Moreover, Non-Radial Pulsations (NRP) can be a crucial explanation of transient mass ejections in Be stars. The classical observational techniques, as photometry and spectroscopy, suffer from the observational selection of NRP modes that is generally impossible to distinguish from physical selection. For example, the observational selection is different for pair and impair modes (integration of symmetric and asymmetric brightness or velocity distributions) and could explain the fact that only pair (or impair) modes are observed in some Be stars, a phenomenon that can also be due to a physical effect as argued by, for example, Jankov et al. (2000). The mechanisms governing the time variations of the mass ejection of Be stars remain largely debated. One possible explanation is the transient combination of several modes of non-radial pulsation (NRP) (Rivinius et al. 1999). It depends on the excited modes, which in turn critically depend on the fundamental stellar parameters (Levenhagen et al. 2003). The stellar diameter, flattening, rotation velocity, differential rotation and gravity govern the dominant excitation mechanisms. Limb and gravitational darkening have a strong impact on the interpretation of time evolution of spectrophotometric data. However, 
differential interferometry yields differential phase information on non resolved objects which allows to measure the diameter, flattening, rotation velocity and differential rotation, and allows much better identification of NRP modes that the spectroscopy and/or photometry alone (e.g. Jankov et al. 2005). Physical selection mechanisms would select equatorial modes if a high latitudinal differential rotation has a destabilizing effect (Stockley \& Buscombe 1987), but the detection of such modes is also favored by observation biases depending from the observation angle. Even if Be stars can be only marginally resolved with the largest VLTI baselines, and structures in the disk are completely unresolved, differential interferometry can extract the displacement with wavelength $(\lambda)$ of the photocenter $\epsilon(\lambda)$ of an unresolved source from the small variations of the interferometric differential phase through a spectral line (Petrov 1988 and Vakili \& Percheron 1991). Recently, we have used this to measure the diameter and the rotation velocity of Achernar (Domiciano de Souza et al. 2012). Jankov et al. (2001) treated explicitly the case of non-radial stellar pulsations. The photocenter shift delivers the first order moment of the spatial brightness distribution and some stellar regions are reinforced. Consequently, the modes that are observationally canceled in flux spectrum should appear in the spectrally resolved photocenter shift. The full reconstruction of the NRP modes requires a Fourier temporal analysis of the photocenter displacement $\epsilon(\lambda, t)$, in a generalization of the Fourier Doppler Imaging based on the spectrum $S(\lambda, t)$.

In this context, long baseline interferometry using spectral resolution in different bands from the visible to the IR, offers new oppotunities to observe the details of such stars with enough spatial resolution (e.g. van Belle 2012) to go beyond the limitation of classical techniques such as spectroscopy, photometry and polarimetry. We hereby describe a numerical model that includes a subset of the different mechanisms explained above: namely fast rotation and stellar pulsation that shape the emergent flux as a function of different parameters such as rotation rate (therefore flatenning), inclination angle to the line of sight, iso-velocity maps across the spectral line among others. The intensity of the differential phase signal critically depends on the characteristics of the observed spectral lines. In HR-K we have access to $\mathrm{Br} \gamma$, which will be strongly polluted by circumstellar emission and to He lines, which are often strongly affected by atmospheric lines and will also be affected by circumstellar emission, but in a way different than for Br.

Our work including the effect of NRP can be innovative, especially with technological advances in interferometry. Observations campaigns on AMBER / VLTI had been requested and obtained for 2013 by our team, in the hope to validate our numerical model.

\section{SCIROCCO+}

\subsection{Theoretical discription of the model}

SCIROCCO+: stands for Simulation Code of Interferometric-observations for rotators and CirCumstellar Objects including non-radial pulsations. It is written 
in Matlab and makes use of the following semi-analytical approach, adopting the frame depicted in Figure 1 (shown in cartesian reference): a pixellized intensity map is computed independently from a velocity map, and both are combined into a spectrally-resolved intensity image-cube, which can be input in a later step into an interferometric simulation code. This model was inspired by an anterior virsion; Simulation Code of Interferometric-observations for rotators and CirCumstellar Objects (SIROCCO) which does not include the non-radial pulsations effects (Hadjara et al. 2012).

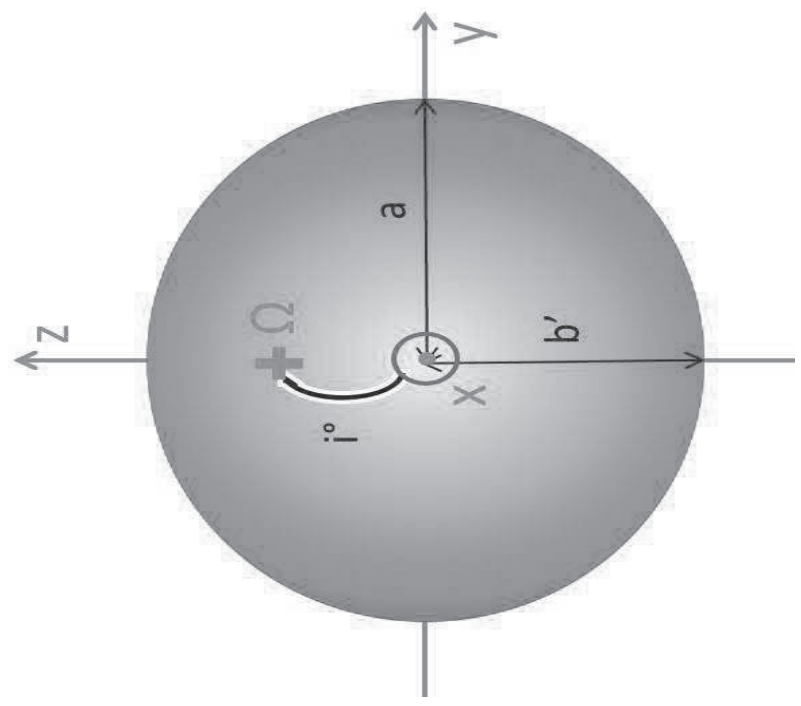

Fig. 1. Adopted reference system for a rotating star (flattened star with major axis $a$ and minor axis $b$, here the apparent minor axis is $b^{\prime}=a b /(a+(b-a) \cos i)$; assuming ellipsoid revolution principal/equations). The cross indicates the point where the rotation axis crosses the stellar surface. This rotation axis forms an angle i with the observer's direction ( $x$ axis) and its projection onto the sky is parallel to the $z$ axis.

\subsubsection{Intensity map}

First, an intensity map of the star's photosphere is computed. We can use for example a simple limb-darkened model from (Hestroffer 1997), expressed in the geographical (co-latitude, longitude) coordinates $(\theta, \phi)$ :

$$
I_{c}(\theta, \phi)=I_{0}\left(1-\epsilon_{\lambda}(1-\mu(\theta, \phi))\right)
$$

where $I_{0}$ represents the flux at the center of the star, $\epsilon_{\lambda}$ is the limb darkening parameter, and $\mu(\theta, \phi)$ is the cosine of the angle between the normal to the surface at the point considered and the observer direction (Domiciano de Souza et al. 2004). The contour of the star is delimited by an ellipse with the minor axis in the 
direction of the rotation axis of the star. The minor-to-major axis depends on the rotation rate following the prescriptions of inclination angle $\mathrm{i}$ (see Fig. 1). $I_{0}$ can serve as a weighting of the continuum flux as a function of wavelength $(\lambda)$ using for example a Planck's law:

$$
I_{0}\left(\lambda, T_{\text {eff }}\right)=\frac{2 h c^{2}}{\lambda^{5}} \frac{1}{e^{\frac{h c}{\lambda \sigma T_{\text {eff }}}}-1}
$$

$h$ being Planck's constant, $c$ the speed of light, $\sigma$ the Boltzmann constant, and $T_{\text {eff }}$ the effective temperature of the star. $I_{0}$ can also be used to input the von Zeipel's effect into our model, by considering a co-latitude-dependent temperature in the below-mentioned local gravity field equation:

$$
I_{0}(\theta) \propto F(\theta)=\sigma T_{\mathrm{eff}}^{4}(\theta)
$$

with $T_{\text {eff }}(\theta) \propto g^{0.25}(\theta), g$ being the local gravity field, also called the modulus of local effective surface gravity $g=|\nabla \Psi(\theta)|$, with $\Psi(\theta)$ is the stellar equipotential surfaces (Domiciano de Souza et al. 2002). An example of intensity map combining rotational flattening and gravity darkening is shown in Figure 2 (top).

\subsubsection{Velocity map}

SCIRROCO + produces a velocity map where we consider rotation and non-radial pulsations:

$$
V_{\text {proj }}(\theta, \phi)=V_{\text {rot }}(\theta, \phi)+V_{\mathrm{nrp}}(\theta, \phi)
$$

In this equation we represent the global velocity map combining rotational flattening and non-radial pulsationsis shown in Figure 2 (bottom).

Where non-radial pulsations velocity has been introduced:

$$
V_{\mathrm{nrp}}(\theta, \phi)=v_{p u l s} * Y_{l m}=v_{\text {puls }} * \sqrt{\frac{(2 l+1)}{4 \pi} \frac{l-|m| !}{l+|m| !}} P_{l,|m|} \cos (\theta) e^{i m \phi} .
$$

With, $v_{\text {puls }}$ : the average velocity pulsation, $l$ : the mode order, $m$ : the mode azimutal order, and $P_{l,|m|}$ : the Legendre function.

And rotational velocity be written:

$$
V_{\text {rot }}(\theta, \phi)=V_{\text {eq }} \cos (\phi)\left(1-\alpha \sin ^{2}(\theta)\right) \sin (i)
$$

Where $V_{\text {eq }}$ represent the equatorial rotation velocity, and the parameter $\alpha$ allows us to include a parametric differential rotation law (Domiciano de Souza et al. 2004).

An example of the non-radial pulsations velocity map is shown in Figure 3 (top) \& another example of pure rotational velocity map is shown in Figure 3 (bottom). 

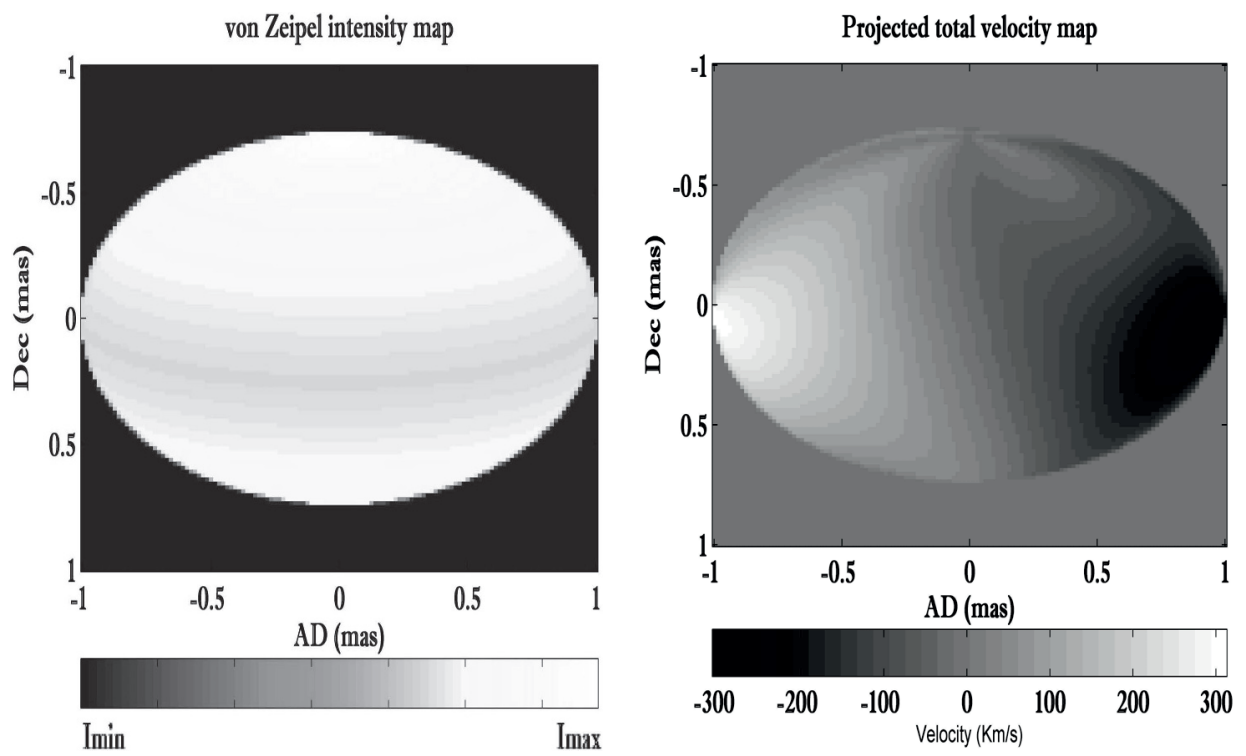

Fig. 2. Left: simulated $\eta$ Centauri intensity map at continuum. The intensity at the poles is greater than at the equator. Here the velocity is upper than $80 \%$ of the critical velocity of the star. Right: global iso-velocity map (rotation+pulsation) of the same model (inclination $57^{\circ}$ ). Here the simulated rotation is differential (the velocity of rotation at the equator is $60 \%$ larger than at the poles).

\subsubsection{Spectrally-resolved image cube}

The last step of the modelization process is to compute $\lambda$-dependent maps. For that, we need to model the natural line-profile of the considered line: we can assume e.g. Gaussian, Lorentzian, or Voigt profile, at the central wavelength $\lambda_{0}$ :

$$
\left\{\begin{array}{l}
H_{\text {Gauss }}(\lambda)=1-H_{0}\left[-\pi H_{0}^{2} \frac{\left(\lambda-\lambda_{0}\right)^{2}}{W^{2}}\right] \\
H_{\text {Lorentz }}(\lambda)=1-\left[\frac{H_{0}}{1+\left(\frac{\lambda-\lambda_{0}}{W / 2}\right)^{2}}\right] \\
H_{\text {Voigt }}(\lambda)=\left(H_{\text {Gauss }} * H_{\text {Lorentz }}\right)(\lambda) .
\end{array}\right.
$$

Where $H_{0}$ and $W$ are the central depths and the equivalent width, respectively. The symbol $*$ represents the convolution product.

The last step calculates the intensity maps of the star as a function of wavelength. For that, we project via the Doppler effect the global velocity map ( $V_{\text {proj }}$, Eq. (2.4)) to the intensity map $\left(I_{c}\right.$, Eq. $\left.(2.1)\right)$, given the line profile $(H$, Eq. $(2.7))$ and the work wavelength $\lambda$ :

$$
I(\lambda, \theta, \phi)=H\left(\lambda+\lambda_{0} \frac{V_{\text {proj }}(\theta, \phi)}{c}\right) I_{c}(\theta, \phi) .
$$



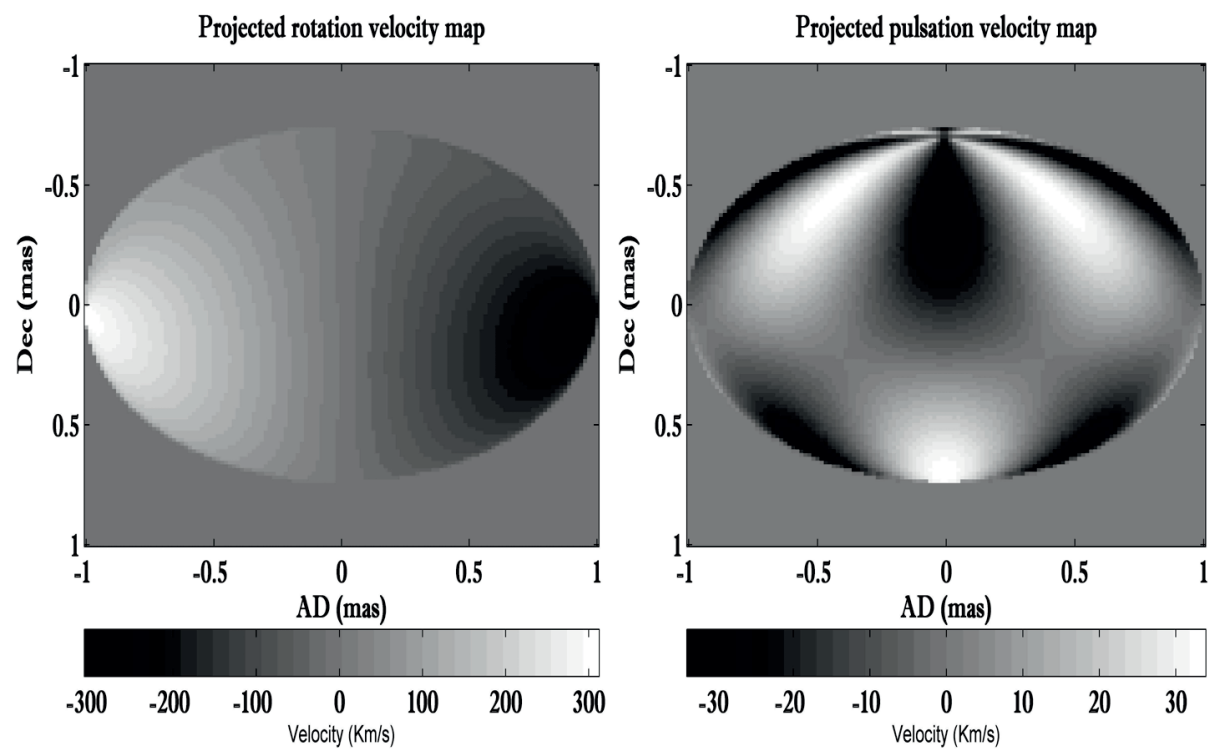

Fig. 3. Left: iso-velocity of pure rotation map (inclination $70^{\circ}, 0^{\circ}$ orientation), the direction of rotation thereby is from the left to the right (from the brightness to darkness). Right: iso-velocity of pure pulsation map, we note that $m=4$, the mode azimuthal order (number of nodes lines that pass through the centers of vibration) and that $l=5$, the mode order (total number of nodes lines). For the both, the color code adopted here, is brightness for the positive velocities and darkness for the negative ones.

We get one intensity map per wavelength of interest around the central wavelength $\lambda_{0}$ of the line (see Fig. 4, left). Once all intensity maps are computed, we synthesize the interferometric observables by Fourier-Transforming each map (see Fig. 4, right). This provides us spectra, visibility amplitudes, phases, and closure phases.

By comparing the observed interferometric measurements to the synthesized quantities, we can access to the parameters of the fast rotating star such as: effective temperature as a function of co-latitude, rotational rate, inclination, angular radius and flattening and, if possible the differential rotation.

\subsection{Interferometric observations simulations - Application to $\eta$ Cen}

Assuming the following characteristics (Table 1):

Table 1. $\eta$ Cen chosen parameters.

\begin{tabular}{||cc|cc|cc||}
\hline \hline Star & Eta Centauri & $v_{\text {puls }}(\mathbf{k m} / \mathbf{s})$ & 34 & $\mathbf{T}_{e q}(K)$ & 16000 \\
Spectral type & $B e$ & Orientation $\left(^{\circ}\right)$ & 0 & $\mathbf{R}_{\text {pole }}(\mathbf{R} \odot)$ & $3-4$ \\
Velocity $v(\mathbf{k m} / \mathbf{s})$ & 340 & Gravity darkening $\beta$ & 0.25 & $\mathbf{R}_{e q}(\mathbf{R} \odot)$ & $5-6$ \\
Inclination i $\left({ }^{\circ}\right)$ & 70 & $\mathbf{T}_{\text {pole }}(K)$ & 21000 & Oblateness & 0.34 \\
\hline \hline
\end{tabular}



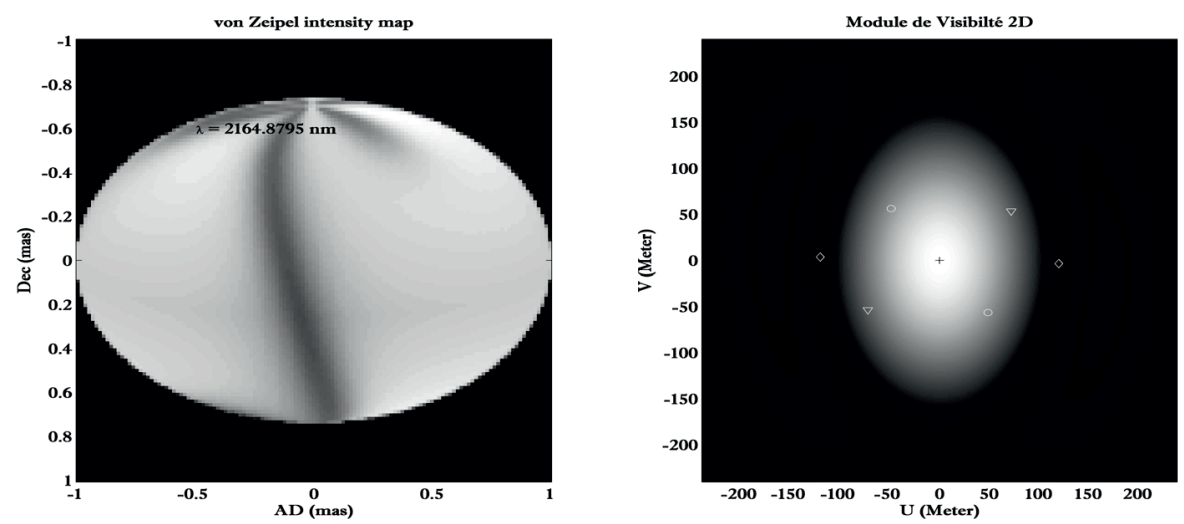

Fig. 4. Left: monochromatic intensity map for a given wavelength. Right: map of corresponding 2D module visibility, which is represented on the three bases with interferometric which will make the observation (1st base small circles, 2nd small triangles and 3rd small diamonds).
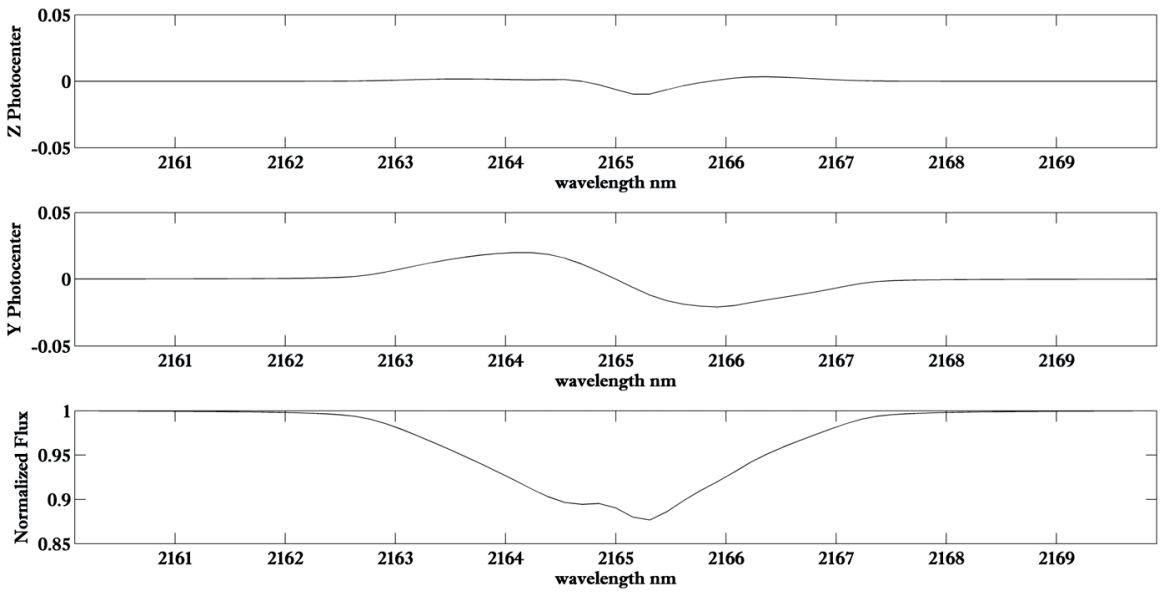

Fig. 5. Top: photo-center (or centroid: the first order term of the phase by Mac Lauren development Jankov et al. 2001) along the $\mathrm{Z}$ axis (see reference adopted in Fig. 1). We note well, here, the influence of the pulsation effect in addition to the inclination effect. Middle: photo-center by Y (note that the photo-centers are in radian). Bottom: normalized spectrum, we see well that our starting line (with a depth of 0.6, and a $F W H M=10 \Delta \lambda$ ) has expanded and its depth was decreased (precisely because of the rotation), it is impacted too by the pulsation (the double hump at the bottom of the spectrum).

In addition, we introduce to our model a differential rotation coefficient $(\alpha=0.6)$ and a Voigt intrinsic line profile with a depth of 0.6 , and a $F W H M=10 \Delta \lambda)$. 

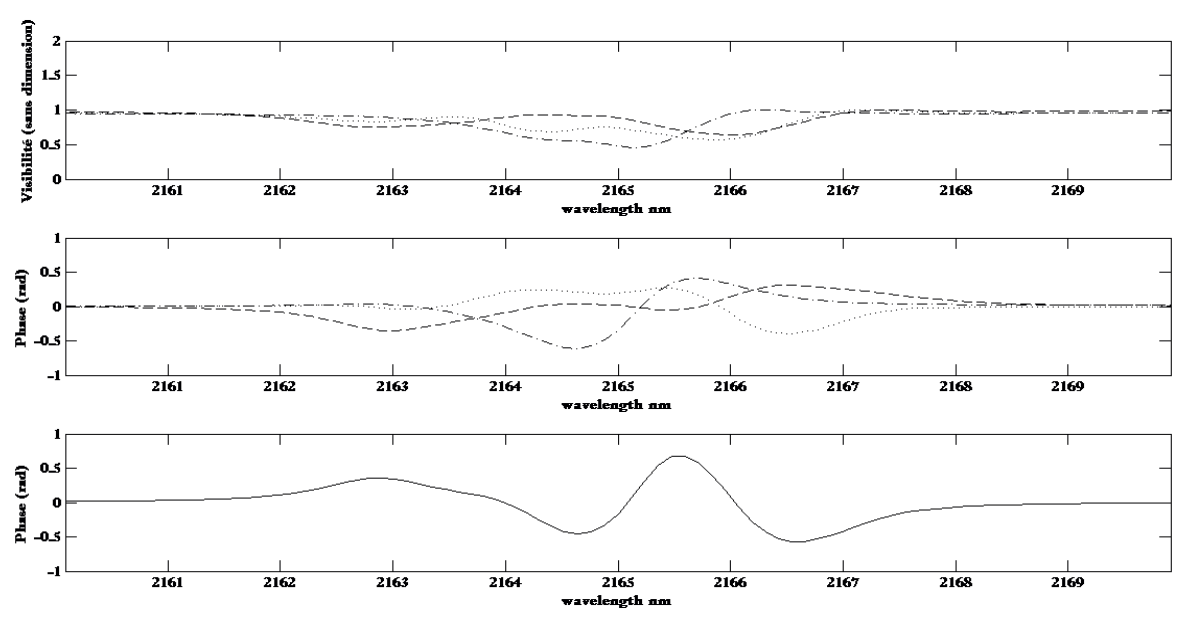

Fig. 6. Top: module visibility observed by the interferometric three bases (dot line for the cercles base, dash-dot line for the triangles base and dash line for the diamonds base). Middle: the phases observed in the three interferometric bases (dot line for the cercles base, dash-dot line for the triangles base and dash line for the diamonds base), we note that the dash-dot line \& the dot line are in opposite phase when their corresponding bases (cercles base \& triangles base) are perpendicular. We note although the phase observed by the base close to perpendicular to the axis of rotation of the star (dash-dot line) is the one that has the highest amplitude \& inversely that which is close to along the axis of rotation (dot line) is low, without forgetting that the dash line is the lowest that the corresponding interferometric base is outside of the first visibility lobe (diamonds base). Bottom: the closure phase; which is classically defined, in AMBER, as: $\Psi=\Phi_{12}+\Phi_{23}-\Phi_{13}$.

We choose to simulate interferometric observations with the AMBER/VLTI instrument on the 3 following interferometric baselines : $K 0-G 1\left(74.63 \mathrm{~m},-139^{\circ}\right.$.), $G 1-A 0\left(90.12 \mathrm{~m},-53.6^{\circ}\right), A 0-K 0\left(120.6 \mathrm{~m},-91.7^{\circ}\right)$, around the Brackett $\gamma$ line $(2.165 \mu \mathrm{m})$. Without forgetting the pulsation parameters of the star: $m=4 ; m$ is the azimuthal order of the mode (number of nodes lines that corss the vibration poles) $\& l=5 ; l$ is the mode order (the total number of nodes lines). The star pulsing with a velocity $v_{\text {puls }}=34 \mathrm{~km} / \mathrm{h}$.

The intensity map at continuum \& global iso-velocity map are shown in Figure 2, the pure iso-velocity rotation map \& pure iso-velocity pulsation map in Figure 3, the monochromatic intensity map at a given wavelength \& corresponding $2 \mathrm{D}$ visibility amplitude map in Figure 4, the photo-centers \& spectrum in Figure 5, and finally visibilities, phases \& closure phase in Figure 6.

\section{Conclusions \& discussions}

We presented here a semi-analytical model of fast-rotators including non-radial pulsations whose aim is to interpret interferometric datasets. We are able to produce interferometric observables using a set of physical parameters like the 
rotation law, gravity darkening effect, etc., while keeping the computing time reasonable (one set of visibility curves can be computed in $17 \mathrm{~s}$ ).

Note that for this simulation, we have obtain the same shape of spectrum (in Fig. 5) as observing by Levenhagen et al. (2003), with spectroscopy in $H \alpha$, which is encouraging.

The next step is to develop a "model-fitting" approach to compare real datasets with this model.

\section{References}

Chelli, A., \& Petrov, R.G., 1995, A\&A, 109, 401

Collins, G.W., 1974, ApJ, 191, 157

Collins, G.W., \& Harrington, J.P., 1966, ApJ, 146, 152

Collins, G.W., \& Sonneborn, G.H., 1977, ApJ, 34, 41

Domiciano de Souza, A., Hadjara, M., Vakili, F., et al., 2012, A\&A, 545, 130

Domiciano de Souza, A., Zorec, J., Jankov, S., Vakili, F., \& Abe, L., 2004, A\&A, 418, 781

Domiciano de Souza, A., Kervella, P., Jankov, S., et al., 2003, A\&A, 407, L47

Domiciano de Souza, A., Vakili, F., Jankov, S., Janot-Pacheco, E., \& Abe, L., 2002, A\&A, 393, 345

Hadjara, M., Vakili, F., Domiciano de Souza, A., Millour, F. \& Bendjoya, P., 2012, SCIROCCO stands for Simulation Code of Interferometric-observations for rotators and CirCumstellar Objects, SF2A 2012, 533

Hestroffer, D., 1997, A\&A, 327, 199

Hutchings, J.B., \& Stoeckley, T.R., 1977, PASP, 89, 19

Hutchings, J.B., Nemec, J.M., \& Cassidy, J., 1979, PASP, 91, 313

Jankov, S., Petrov, R., Vakili, F., Robbe-Dubois, S., \& Domiciano, A., 2005, PASRB, 5, 83

Jankov, S., Vakili, F., Domiciano de Souza, A., \& Janot-Pacheco, E., 2001, A\&A, 377, 721

Jankov, S., Janot-Pacheco, E., \& Leister, N.V., 2000, ApJ, 540, 535

Levenhagen, R.S., Leister, N.V., Zorec, J., et al., 2003, A\&A, 400, 599

Maeder, A., \& Peytremann, E., 1972, A\&A, 21, 279

Martayan, C., Frémat, Y., Hubert, A.-M., et al., 2006, A\&A, 452, 273

Meynet, G., 2009, Lect. Notes Phys., 765, 139

Petrov, R.G., 1988, Diffraction-Limited Imaging with Very Large Telescopes, ed. D.M.

Alloin \& J.M. Mariotti (Kluwer), 249

Rivinius, Th., Štefl, S., \& Baade, D., 1999, MNRAS, 227, 801

Stockley, T.R., \& Buscombe, W., 1987, MNRAS, 227, 801

Vakili, F., \& Percheron I., 1991, Rapid Variability of OB-Stars: Nature and Diagnosis

Value, ed. D. Baade, 15-17 Oct. (ESO, GArching, Germany), 77

van Belle, G.T., 2012, A\&ARv, 20, 51

von Zeipel, H., 1924, MNRAS, 84, 665 